\section{REGENERACIÓN URBANA: UN PANORAMA LATINOAMERICANO}

\section{Resumen}

Tradicionalmente, en el campo del urbanismo, el término regeneración urbana refiere al proceso de producción urbana que se ha vuelto predominante en las ciudades de Europa y América del norte desde hace ya varias décadas: la implementación de grandes proyectos urbanos de recuperación, tanto física como socio-económica, de áreas intra-urbanas, generalmente en desuso. Esto se está dando todavía poco en las ciudades latinoamericanas, a pesar de la existencia de un alto potencial de suelo posible de reciclar. Los proyectos realizados, escasos, son cuestionados. A su

\section{URBAN REGENERATION: A LATIN AMERICAN OVERVIEW}

Catherine Paquette Vassalli

\section{Abstract}

Traditionally, in the field of urbanism, the term urban regeneration refers mainly to the process of urban production that has become dominant in European and North American cities for several decades now: the implementation of large urban projects of both physical and socioeconomic recovery in usually vacant intra-urban areas. Despite the existence of a large potential of land able to be recycled, this has still been happening sparsely in Latin American cities. The projects that have been completed, few, have been called into question. In turn, the programs to revitalize the historic centers and re-densify 
vez, los programas para revitalizar los centros históricos y redensificar las áreas peri-centrales no pueden ser considerados como iniciativas de regeneración urbana, en el sentido en que consistieron principalmente en impulsar dinámicas de puesta en valor turísticocultural y de desarrollo inmobiliario intensivo, respectivamente. Sin embargo, sería un error concluir que no existen experiencias valiosas de regeneración urbana en la región. Éstas se encuentran, aunque bajo otras modalidades, en los barrios populares de las periferias urbanas, donde se están llevando a cabo programas de mejoramiento urbano integral. Descentrar la mirada hacia estas iniciativas podría contribuir a replantear la visión que se tiene de la regeneración urbana en la región.

PALABRAS CLAVES: REGENERACIÓN URBANA, CENTROS HISTÓRICOS, REDENSIFICACIÓN, MEJORAMIENTO DE BARRIOS, GRANDES PROYECTOS URBANOS.

Recibido: 2020-05-18

Aceptado: 2020-09-11 peri-central areas cannot be seen as initiatives for urban regeneration, in the sense that they consisted mainly in promoting dynamics that highlight tourism and culture, and intensive real estate development, respectively. Nevertheless, to conclude that there are no valuable experiences of urban regeneration in the region would be an error. These are found under different modalities, and rather in peripheral workingclass neighborhoods, where comprehensive urban improvement programs are being carried out. Decentering the gaze towards these initiatives may contribute in reassessing the general vision towards urban regeneration in the region.

\section{KEYWORDS: URBAN REGENERATION, HISTORICAL CITY CENTERS, RE-DENSIFICATION, NEIGHBORHOOD IMPROVEMENT, LARGE URBAN PROJECTS.}

Received: 2020-05-18

Accepted: 2020-09-11

Investigadora IRD Institut de Recherche pour le Développement, CESSMA - Centre d'Etudes en Sciences Sociales sur les Mondes Africains, Américains et Asiatiques; Université de Paris; INALCO Institut national des langues et civilisations orientales, Francia, https://orcid.org/0000-0003-33176259. Correo electrónico: catherine.paquette@ird.fr 


\section{Introducción}

Desde hace ya más de una década, las autoridades nacionales y locales de Latinoamérica han estado haciendo la promoción activa de un modelo de ciudad más denso y compacto para alcanzar los objetivos de la sostenibilidad urbana. Basta con revisar rápidamente el contenido de sus políticas urbanas actuales, así como las principales directrices de los planes y programas de desarrollo urbano y ordenamiento territorial vigentes, para darse cuenta que, desde Chile a México, se trata de una nueva prioridad indiscutible a nivel regional. En una región muy influenciada por las agendas internacionales de desarrollo sostenible, la adopción reciente de los objetivos del desarrollo sostenible (en particular del ODS11 dedicado a las ciudades y los asentamientos humanos), y más aún, de la Nueva Agenda Urbana, ha dado un impulso adicional a los imperativos de controlar la expansión urbana y promover ciudades más densas, socialmente vibrantes e inclusivas (CEPAL, 2018).

Los procesos de regeneración urbana suelen ocupar un lugar central dentro de las estrategias enfocadas a la sostenibilidad urbana. Además de ayudar en principio a contener la expansión periférica, la reconstrucción de la ciudad sobre sí misma, como principio genérico, permite optimizar los servicios y equipamientos existentes, limitar los traslados cotidianos (y por lo tanto la contaminación y las emisiones) y favorecer la mezcla de usos, intensificando así la vida urbana.

La implementación de la regeneración urbana parece especialmente oportuna para el caso de las ciudades latinoamericanas, cuya característica común es contar con áreas centrales deterioradas y afectadas por procesos de despoblamiento y desuso (Audirac, 2014). Sin embargo, es preciso reconocer que en la región existe un desfase importante entre las intenciones existentes en la materia y la realidad muy concreta del urbanismo y del desarrollo urbano. Por una parte, los grandes proyectos urbanos de reciclaje no han llegado a constituir realmente una tendencia marcada del urbanismo en Latinoamérica -a diferencia de lo ocurrido en las ciudades de Europa y América del Norte-; por otra, es posible afirmar que los esfuerzos (numerosos) desplegados en la región para revitalizar los centros históricos y las experiencias (más escasas) de redensificación/ repoblamiento de las áreas pericentrales no pueden ser asimilados a procesos de regeneración urbana como tal.

En efecto, como se explicará más adelante, los planes de revitalización para los centros históricos generalmente no incluyen proyectos urbanos de restructuración físico-espacial mayor (componente siempre presente en los proyectos de regeneración urbana) y losesfuerzos se concentran más bien en la recuperación de los espacios públicos, el fomento del turismo y un cambio de imagen. En cuanto a los 
programas de redensificación/repoblamiento, aparecen demasiado centrados en la dimensión habitacional y les falta acompañar el proceso de reinversión inmobiliaria con acciones de mejoramiento del espacio urbano y enmarcarlo en una visión urbanística.

Sin embargo, sería equivocado concluir que la regeneración urbana, como proceso deliberado que busca transformar la ciudad existente en lo físico, lo social y lo económico, no es parte de la actualidad del urbanismo en las ciudades latinoamericanas. En realidad, descentrando la mirada, es posible constatar que está muy arraigada en la región, pero mediante iniciativas que tienen otra denominación. América Latina posee en particular una experiencia valiosa en materia de mejoramiento integral en barrios y asentamientos populares periféricos, que se ha venido consolidando a lo largo de ya varias décadas.

A partir del análisis de fuentes secundarias y de abordajes previos sobre grandes proyectos y estrategias urbanas para centros históricos y áreas centrales en América Latina, este artículo constituye un intento de realizar un balance crítico acerca de la situación de las ciudades de la región en cuanto a lo que constituye una de las tendencias mayores del urbanismo contemporáneo y una preocupación creciente de los gobiernos nacionales y locales. De manera paradójica, aunque el estudio de la gentrificación, como impacto de las intervenciones en materia de regeneración urbana, se ha desarrollado mucho en América Latina a lo largo de la última década (Betancur, 2014), pocas reflexiones han sido llevadas a cabo sobre el proceso de producción urbana que en sí estaría generando éste cambio urbano-social.

La opción de enfocarnos en la temática con una pretensión de análisis regional está siendo asumida plenamente, al igual que sus limitaciones inevitables. Por múltiples razones, más allá de sus especificidades, las ciudades latinoamericanas cuentan con numerosas características comunes, tanto en sus configuraciones urbanas como en las políticas que están siendo implementadas en ellas. Si bien son "ciudades diversas", comparten indudablemente una "narrativa común" (Sedrez, 2013), en particular en materia de urbanismo y de desarrollo urbano (Paquette, 2014).

\section{La regeneración urbana en su sentido urbanístico tradicional}

El término regeneración urbana está siendo usado en un universo cada vez más extendido de ámbitos profesionales ligados a las temáticas urbanas, como en la sociología y las ciencias políticas. Sin embargo, se trata de una noción inicialmente propia del urbanismo, cuya principal característica es no contar con una 
definición rigurosa que permita delinear precisamente sus contornos. Lo anterior se debe, en buena medida, a que el urbanismo no es ciencia ni técnica, sino que pertenece al campo de la acción: se trata ante todo de una praxis (Merlin, 2015). Por lo cual, las nociones del urbanismo se caracterizan generalmente por ser definidas ante todo de manera empírica y no de forma a priori. C. De Magalhães no expresa sino esto cuando precisa, en su intento de definición, que la regeneración urbana no está "firmemente anclada en un marco teórico sólido", sino que se trata de un "campo empírico de política pública que busca atender una problemática urbana especifica [...] sobre la base de intervenciones que han funcionado en circunstancias parecidas" (De Magalhães, 2015).

El término regeneración urbana es originalmente anglosajón. Apareció en Gran Bretaña durante la segunda mitad del siglo XX para referirse a intervenciones urbanísticas realizadas para reconquistar los grandes baldíos industriales y portuarios. Su adopción en la literatura científica es bastante reciente. En los trabajos existentes, la regeneración urbana stricto sensu suele ser definida como un nuevo proceso de producción urbana que surgió hace ya varias décadas, en particular a partir de los años 80. Este, que consiste en la realización de grandes proyectos urbanos de reconversión de la ciudad existente, se ha vuelto "la tendencia mayor del urbanismo contemporáneo" en las ciudades de Europa y América del Norte (Rodrigues-Malta, 2001). De manera general, en estas ciudades, las iniciativas públicas se han volcado vigorosamente hacia la reutilización del potencial intra-urbano, abandonando así, y desde los 70, la planificación de grandes proyectos de urbanizaciones nuevas que prevalecían anteriormente (Chaline, 1996)².

El carácter novedoso de la regeneración urbana, como proceso de reciclaje urbano, podría a priori ser cuestionado. En efecto, desde que existen las ciudades, la sustitución y adecuación funcional de los espacios y de los inmuebles siempre han ritmado la transformación de la forma urbana. Sin embargo, lo que sucedió en las ciudades europeas y norteamericanas a partir de mediados del siglo XX se dio a una escala totalmente inédita: a raíz de la salida masiva de las instalaciones industriales y portuarias, se desocuparon vastas superficies de suelo intra-urbano, muchas veces con ubicaciones céntricas, a las cuales las autoridades tuvieron que encontrar otro uso. El ejercicio de reciclaje cambió por lo tanto de ritmo y de naturaleza, generando la puesta en marcha generalizada de políticas llamadas de regeneración urbana (Rodrigues-Malta, 2001).

2 Si bien no se detuvo para nada el desarrollo urbano en periferias, éste fue más bien, a partir de entonces, el resultado de iniciativas privadas por parte de inmobiliarias, y no de grandes proyectos impulsados por las autoridades, como solía ser anteriormente. 
La escala en los procesos de reciclaje no es la única característica de la Regeneración Urbana. A pesar de la ausencia de una definición precisa del término, existe un consenso implícito acerca de lo que implicaría esta nueva forma de producción urbana. La regeneración urbana forma parte de la familia de los ahora numerosos términos urbanísticos iniciados con el prefijo "re" (revitalización, reconversión, rehabilitación, recualificación, recomposición) que muestran claramente la orientación del urbanismo hacia los procesos de reconstrucción de las ciudades sobre sí mismas (RodriguesMalta, 2001). Sin embargo, la regeneración urbana difiere de la renovación urbana (urban renewal) en el sentido en que no busca sólo un proceso de cambio físico-espacial, sino también una dinamización económica, mediante la atracción de nuevas actividades económicas y de residentes y un cambio de imagen global ${ }^{3}$. Se distingue también de la revitalización urbana o de la rehabilitación urbana en el sentido en que estas acciones no implicarían ningún modo de acción específico. En cambio, la regeneración urbana consiste en abordar el reciclaje urbano

3 Si bien es cierto, como lo demostró por ejemplo el trabajo icónico de H. Coing sobre la renovación urbana en Paris, que la renovación urbana siempre conlleva impactos sociales importantes (Coing, 1966), éstos no forman parte de los objetivos explícitos y tampoco corresponden al resultado de acciones específicas implementadas para generar esta transformación. La recomposición social es por lo tanto un resultado (al parecer, inevitable) y no un objetivo explícito y revindicado de las intervenciones, a diferencia de lo que sucede con la regeneración urbana. buscando al mismo tiempo una transformación física, económica y social y activando para esto, por lo tanto, diversas palancas. Esto se daría además en una visión de largo plazo y con un modo de acción estratégico, orientado hacia un objetivo muy preciso (Roberts, 2008).

Otra característica destacable de la regeneración urbana tiene que ver con que los gobiernos locales casi siempre están en el origen de las iniciativas en la materia, inclusive impulsando a veces la construcción de grandes equipamientos culturales emblemáticos, como son los museos (Chasseriau, 2002). Sin embargo, los proyectos suelen al mismo tiempo, involucrar de manera estrecha a inversionistas privados. De hecho, el carácter multi-actoral y la asociación público-privado en particular, casi parece ser parte integral de la definición implícita de la Regeneración Urbana: debido a la envergadura de los proyectos, se requiere de importantes recursos financieros que los gobiernos locales no poseen (Amirtahmasebi, Orloff, Wahba y Altman, 2016). Resulta interesante observar que esta última característica de la regeneración urbana es la que mayor crítica genera hacia este proceso en América Latina. Los investigadores tienden a ver en los grandes proyectos urbanos de este tipo la manifestación por excelencia del protagonismo creciente de los intereses económicos privados en el proceso de producción de la ciudad y el nuevo rol de las autoridades como "socios institucionales" del sector privado, promoviendo un "urbanismo 
ligado a los promotores" (Cuenya, 2012; Jajamovich, 2012; Kozak y Feld, 2018) ${ }^{4}$.

La práctica de la regeneración urbana se ha diversificado mucho desde los años 1980, abarcando una gran variedad de proyectos. Sin embargo, las características antes mencionadas siguen formando parte de la principal definición implícita del término en el campo del urbanismo, aunque, como veremos adelante, las acciones llevadas a cabo para rehabilitar los grandes conjuntos de vivienda social deteriorados constituyen el otro sentido, secundario, que se le está dando a la regeneración urbana en los países del Norte.

A la luz de los elementos mencionados previamente, y en un esfuerzo por sintetizar lo que abarcaría el término de regeneración urbana, se podría por lo tanto proponer, a modo de una definición genérica propia, que esta noción se refiere por lo general a proyectos urbanos de escala significativa (generalmente barrial), impulsados por los gobiernos locales y en asociación estrecha con actores privados, cuyo objetivo es reconvertir porciones de la ciudad existente, transformándolas, a diferencia de la renovación urbana y de la revitalización urbana, al mismo tiempo en lo físico, lo social

$4 \quad$ Llamalaatención notar que la percepción negativa que se tiene de los proyectos de regeneración urbana en Latinoamérica, en particular por parte de la academia, no existe con la misma magnitud en Europa, donde estas intervenciones, mucho más estudiadas, están vistas de manera más neutral (Aureau, Dufourmantelle y Narring, 2015. y lo económico; buscando además cambiar su imagen para atraer nuevos residentes, actividades y usos.

\section{Los grandes proyectos de regeneración urbana en las ciudades latinoamericanas: ¿un campo rezagado?}

Si bien se han dado en la región varias experiencias significativas en materia de Grandes Proyectos Urbanos (GPU) de recuperación de espacios intra-urbanos en desuso o desaprovechados, éstas no son numerosas, sobre todo en comparación con lo que sucedió en Europa y América del Norte y en relación al potencial de suelo urbano por reciclar que existe en las ciudades latinoamericanas. Por el momento, se puede afirmar que, en comparación con las ciudades del Norte, la región se ha quedado atrás en materia de regeneración urbana (Betancur, 2014).

Refiriéndonos a los GPU en Latinoamérica, resulta importante precisar que la acepción del término es muy amplia: es así como se incluyen generalmente bajo este concepto a los nuevos sistemas de transportes de tipo bus rapid transit y a las infraestructuras viales importantes (Jajamovich, 2019; Lungo, 2005). Este tipo de megaproyectos impactan por cierto directamente a las ciudades, pero no son 
urbanísticos como tales (Paquette, 2014) . De $^{5}$ la misma manera, se llega a considerar a las iniciativas de desarrollo económico local como Proyectos de Regeneración Urbana ${ }^{6}$. Ahora bien, si nos limitamos a considerar los proyectos cuyo objeto central es la transformación del tejido urbano edificado, podemos constatar que, hasta el momento, éstos han sido escasos en las ciudades de la región. El universo aparece aún más reducido si, siguiendo la definición previamente presentada de lo que sería la regeneración urbana, descartamos a las grandes intervenciones urbanas modernizadoras (meramente físicas) de renovación urbana que han sido realizadas en varias ciudades durante la segunda mitad del Siglo XX.

Dentro de los grandes proyectos urbanos de regeneración urbana realizados en América

5 Una definición interesante del concepto de GPU está siendo proporcionada por un informe realizado sobre esta temática en Europa por el Consejo General del Medio Ambiente y del Desarrollo Sostenible (CGEDD, Francia). Según los autores, la definición de un GPU está sujeta a controversias, pero ésta podría corresponder a "un tipo de intervención que difiere de los proyectos urbanos tradicionales debido a la existencia de retos urbanísticos y territoriales a la escala de las metrópolis o de sus áreas de influencia, a su visión integral y cualitativa en el plano ambiental, económico y social, así como arquitectónico y urbano, a su escala y a sus plazos, largos, de realización". Además, un GPU moviliza recursos muy importantes y convoca la asociación de actores múltiples, los que generalmente van más allá del territorio objeto del proyecto" (Aureau et al., 2015).

6 Ver el reciente llamado a proyectos del BID, LAC Cities Challenge: Proyectos innovadores de Regeneración Urbana https://www.iadb.org/es/desarrollo-urbano-y-vivienda/lac-citieschallenge-proyectos-innovadores-de-regeneracion-urbana
Latina, una de las intervenciones más representativa es Puerto Madero, en la ciudad de Buenos Aires (Jajamovich, 2012, 2016). Se trata de una operación urbanística de reconversión de gran escala, con un propósito no sólo físico-espacial sino también económico y social (generar una nueva centralidad aliando actividades económicas terciarias y vivienda de alto estándar), impulsada por parte de las autoridades en asociación con el sector privado y operada por una corporación de desarrollo mixta creada para el propósito. El proyecto, desarrollado con la colaboración de la ciudad de Barcelona, es la experiencia de GPU más estudiada por los investigadores interesados en la temática en Latinoamérica. Mientras genera importantes críticas por parte de la Academia, por aparecer como una clara manifestación del control de los intereses privados sobre el proceso de producción urbana (Cuenya, 2012), recibe, en cambio, fuertes elogios por parte del Banco Mundial, quien celebra el carácter exitoso de la experiencia (Amirtahmasebi et al., 2016).

Yéndonos a experiencias más recientes, destaca en particular el caso emblemático del barrio denominado Nuevo Polanco en la Ciudad de México. A raíz de la inversión que realizaron varios empresarios, encabezados por el multimillonario mexicano Carlos Slim, una zona de antiguas fábricas, colindante con uno de los sectores más codiciados de la ciudad, se ha transformado a lo largo de 
la última década en una centralidad mixta de mayor rango, con edificios corporativos, torres de departamentos de lujo de acceso controlado, centros comerciales, así como museos y equipamientos culturales privados. Marcado por un carácter vertical que contrasta fuertemente con su entorno urbano, el Nuevo Polanco constituye hoy en día una de las zonas con mayor desarrollo inmobiliario de la Ciudad de México, con un incremento poblacional rápido y una gentrificación evidente (Aguayo, 2016). Sin embargo, a diferencia de lo que fue el mega-proyecto Santa Fe en esa misma ciudad, planificado por el Gobierno local y desarrollado por una empresa pública de carácter públicoprivado a partir de inicios de los años 1990 (Valenzuela, 2007), el Nuevo Polanco no es para nada una iniciativa impulsada por las autoridades públicas. Su dinámica fue detonada únicamente por actores privados (por cierto, en el contexto de una clasificación del sector como zona con potencial de desarrollo en el Programa General de Desarrollo Urbano de la ciudad aprobado en 1997), sin que existiera un Plan Maestro elaborado por parte del Gobierno local para esta zona. Lo anterior explica en gran parte los disfuncionamientos urbanos mayores que aparecieron a la brevedad en la zona (colapso de las vialidades existentes, ausencia de espacios públicos). Estos llevaron a las autoridades a detener un tiempo el otorgamiento de licencias de construcción para nuevos proyectos, mientras se veían obligadas a elaborar a posteriori un Plan Maestro para el desarrollo de la zona. Considerando lo anterior, este ejemplo se asemeja más a lo que sería un mega proyecto inmobiliario que a una operación de regeneración urbana. El caso no es anodino e ilustra un riesgo importante: el que los proyectos de reciclaje urbano de gran escala, escasos hasta ahora en América Latina, empiecen a detonarse por iniciativa y bajo el control de actores privados, sin participación de las autoridades para su orientación y su conducción. Aunque la participación del Gobierno local no constituye en sí ninguna garantía de que el proyecto urbano cumpla con objetivos de interés público, se trata de una condición indispensable para que esto pueda suceder.

Los factores que han sido identificados como detonadores para el surgimiento de los grandes proyectos urbanos, son generalmente los mismos que permiten explicar por qué no se han dado grandes intervenciones de regeneración urbana en América Latina. Una demanda importante de las empresas globalizadas por aglomerarse en espacios que les garanticen condiciones adecuadas de funcionamiento y visibilidad -a escala planetaria y regional-, sería uno de ellos. La existencia de patrones de consumo por parte de los grupos de muy altos ingresos, orientados hacia espacios exclusivos centrales donde ellos quieran vivir, trabajar y recrearse, sería otro elemento determinante (Cuenya, 2012). Existe respecto a este último factor una diferencia fundamental entre los contextos 
urbanos de Europa/América del Norte y América Latina. La demanda por parte de los hogares de la elite globalizada por residir en los "nuevos espacios urbanos de la globalización" es mucho más reducida en América Latina. Más aún si estamos hablando de las áreas urbanas centrales recuperadas, con las cuales estos grupos de población mantienen una relación compleja, marcada en particular por un rechazo importante (Betancur, 2014). Además, se están desarrollando todavía en Latinoamérica grandes proyectos urbanos en zonas ubicadas fuera o en el límite inmediato de la ciudad, sea para hogares de clase media-alta o alta, bajo la forma de urbanizaciones cerradas, o para estratos bajos (Hidalgo, Borsdorf y Zunino, 2008; Libertun, 2017; Valenzuela, 2007).

\section{Los programas de revitalización y/o repoblamiento en las áreas céntricas}

Los grandes proyectos urbanos de reconversión intra-urbana son todavía escasos en Latinoamérica. En cambio, numerosas ciudades de la región han desarrollado planes para revitalizar sus centros, en particular cuando éstos cuentan con un patrimonio histórico importante. Además de acciones en centros históricos, se han implementado en algunas metrópolis programas de redensificación y repoblamiento de las áreas centrales, tratándose aquí de espacios más amplios que los meros centros y que corresponden a los primeros contornos urbanos históricos. Estas acciones impulsadas por las autoridades locales se han denominado como revitalización o rehabilitación (generalmente integral), en el caso de los centros históricos, y redensificación y repoblamiento cuando se trata de las áreas centrales. A pesar de que están siendo desarrolladas en espacios urbanos especialmente aptos para detonar proyectos de regeneración urbana, y que comparten elementos en común (tal como la participación del sector privado y un impulso por parte de los gobiernos locales), no pueden ser asimiladas a este tipo de intervención sobre la ciudad.

\section{REVITALIZACIÓN DE CENTROS HISTÓRICOS: LAS CIUDADES LATINOAMERICANAS, EN PRIMERA LÍNEA}

Desde hace ya por lo menos tres décadas, numerosos planes de acción han sido emprendidos en América Latina por los gobiernos locales para detonar la revitalización y la rehabilitación de los centros históricos. Estas iniciativas generaron una bibliografía extremadamente amplia, compuesta por trabajos de investigación, tesis de estudiantes, así como por informes realizados por expertos 
en la temática ${ }^{7}$. Si bien los planes elaborados para los centros históricos han sido presentados como integrales, en el sentido en que intentan cubrir todas las áreas sectoriales que implican las políticas urbanas, la realidad de las acciones impulsadas es otra. Aparte de la restauración de edificios patrimoniales (que no es una medida urbana como tal), los objetivos perseguidos en los planes de acción suelen estar muy enfocados en la recuperación de los espacios públicos: incluyen la remodelación de plazas y banquetas, la peatonalización de calles, la ineludible reubicación de vendedores "ambulantes" (que permite "liberar" estos espacios) y medidas para generar seguridad (Cabrera, 2019; Crossa, 2009; Pineda y Velasco, 2017; Stamm, 2008). Estas acciones, que se aplican también en los nuevos distritos culturales desarrollados en barrios tradicionales como La Boca en Buenos Aires o Bellavista en Santiago de Chile, no están dirigidas hacia los propios residentes y apuntan más bien al desarrollo del potencial turístico y cultural de la zona (González Couret, 2015; Navarrete, 2017). Si bien la dimensión habitacional constituye una de las principales problemáticas de los centros históricos de la región, reconocida por los gobiernos locales y mencionada dentro de los ejes estratégicos de muchos planes de revitalización, ésta sigue siendo objeto de pocas acciones concretas, sea

$7 \quad$ Una bibliografía reciente está proporcionada por Betancur en su trabajo de análisis de la gentrificación en la Región (Betancur, 2014) para mejorar el parque habitacional existente o para generar una oferta nueva, popular o de mayor nivel. Por cierto, se han realizado proyectos valiosos de mejoramiento de vivienda popular (Delgadillo, 2008), pero éstos han sido puntuales. Las experiencias implementadas en materia de vivienda, por ejemplo, en la Ciudad de México a principios de los años 2000 o también en Quito durante el mismo periodo, no han dado resultados significativos. Hoy en día, a pesar de varias décadas de intervenciones, el tema sigue siendo uno de los ángulos muertos dentro de las acciones implementadas en los centros históricos (Carrión, 2017). Esto se debe, sin lugar a dudas, a que la problemática es altamente compleja y que se acumulan un sinnúmero de dificultades y frenos a una recuperación habitacional: la presencia de numerosos edificios y predios intestados; los precios generalmente muy elevados de las propiedades susceptibles de ser cedidas; las limitaciones impuestas por los organismos responsables de catalogar y proteger el patrimonio histórico; las resistencias por parte de los grupos que viven o trabajan en los centros históricos, y la imagen de estos espacios, centralidades populares que generan un rechazo por parte de las clases medias y media-altas que los gobiernos locales quisieran atraer, entre otros.

Lo anterior explica que lo que ha sucedido en los centros históricos de las ciudades latinoamericanas difícilmente pueda 
asemejarse a un proceso de regeneración urbana como tal, que implicaría contar con todo un abanico de objetivos y acciones mucho más amplio y completo. La ausencia de grandes proyectos urbanos, es decir de intervenciones urbanísticas significativas que generen una transformación físico-espacial del tejido urbano, es otra característica común de los procesos de revitalización y rehabilitación de los centros históricos, que lleva a cuestionar su posible pertenencia a lo que sería un proceso de regeneración urbana. El proyecto Alameda, en el centro histórico de la Ciudad de México, es uno de los pocos que se podría mencionar como un ejemplo en la materia (Hernández Cordero, 2012).

\section{REDENSIFICACIÓN/REPOBLAMIENTO DE ÁREAS CENTRALES: ¿REGENERACIÓN URBANA O DESARROLLO INMOBILIARIO INTENSIVO?}

El despoblamiento ha sido un rasgo común de las áreas centrales de las principales ciudades latinoamericanas durante la segunda mitad del siglo XX (Audirac, 2014). Para contrarrestar este proceso, dos metrópolis en particular decidieron implementar una estrategia ambiciosa de repoblamiento y redensificación, basada en el regreso de la construcción habitacional nueva en sus áreas centrales.

La primera experiencia fue la del Municipio de Santiago, en el corazón del Área Metropolitana de Santiago de Chile. Entre 1952 y 1992, debido a una serie de causas características del proceso de declive de las áreas centrales de las ciudades en la región, el municipio había perdido la mitad de su población residente y el 33\% de su parque habitacional. Al inicio de los años 1990, las autoridades municipales lanzaron el Programa de Repoblamiento de Santiago Centro, un ambicioso programa de renovación urbana cuyo objetivo era incrementar la población municipal en 100.000 habitantes en un lapso de 15 años, gracias a un regreso significativo de la oferta de vivienda nueva en altura destinada ante todo a la clase media (Contreras, 2011). La segunda experiencia destacada en la región fue la que puso en marcha en México el ex Distrito Federal (ahora Ciudad de México) en 2002, para la redensificación de la Ciudad Central, espacio compuesto por las cuatro delegaciones administrativas y políticas centrales, cuya población residente se había reducido también drásticamente a lo largo de tres décadas. Al igual que en Santiago de Chile, se trataba de reorientar la construcción de vivienda nueva colectiva hacia la Ciudad Central, área de la ciudad abandonada por los promotores inmobiliarios (Delgadillo, 2016).

Las dos experiencias difirieron de manera importante. En el caso de Santiago de Chile, la autoridad municipal jugó un papel muy proactivo durante la primera fase del Programa (primera década), impulsando el regreso de la construcción habitacional nueva a través de la Corporación para el desarrollo de Santiago 
(entidad mixta público-privada), identificando las propiedades potenciales de recuperar, relacionando sus dueños con desarrolladores y convenciendo, además (y sobre todo), al Ministerio de la Vivienda y Urbanismo de crear, dentro del marco de la política habitacional nacional vigente, un Subsidio de Renovación Urbana que resultó ser decisivo para subsidiar la demanda (Amirtahmasebi et al., 2016). En cambio, el Gobierno del Distrito Federal no demostró tanto protagonismo para detonar el proceso de redensificación: laestrategiaconsistió más bien, mediante un "bando" de Gobierno (el llamado Bando 2), en dejar de autorizar las construcciones nuevas de viviendas en edificios en las demarcaciones periféricas de la ciudad, hasta entonces las más atractivas, y al contrario, en agilizar los trámites legales para la realización de proyectos inmobiliarios en la Ciudad Central, gracias a la creación de una ventanilla única (Tamayo, 2007). Las dos experiencias fueron sin embargo muy similares, tanto en su filosofía como en sus impactos. En ambos casos, el principio rector consistió en dejar el repoblamiento/la redensificación totalmente en manos de la inversión inmobiliaria privada, con un marco de actuación sumamente abierto y flexible, sin plantear ninguna visión urbana ni urbanística. No se estableció ningún tipo de lineamiento precisando el tipo de "imagen urbana" que se pretendía promover. Tampoco se definió en qué sectores se iba a privilegiar la redensificación y en cuáles no, tomando en cuenta las características físicas y sociales de los distintos barrios y finalmente, la "capacidad de carga" (Cortés Chavez, 2009) de los distintos micro-territorios para soportar un proceso de densificación. Tampoco se articuló la estrategia de repoblamiento/redensificación con otras políticas urbanas sectoriales, como ocurrió en el Distrito Federal, en México, en materia de transporte y movilidad (Paquette, 2008).

En el caso de Ciudad de México, se ha calificado el proceso de redensificación como un "desarrollo urbano intenso" (Delgadillo, 2016). Sin embargo, hablar de "desarrollo inmobiliario intenso" podría ser hasta más oportuno, precisamente porque la estrategia impulsada no conllevó ninguna dimensión urbanística y consistió exclusivamente en detonar un boom inmobiliario descontrolado. Éste generó externalidades negativas importantes como, entre otras, un alza de los precios del suelo y de los bienes inmobiliarios, inclusive en barrios tradicionalmente populares, con consecuencias en materia de evicción pasiva y paulatina de su población residente (Delgadillo, 2016). También surgió un rechazo marcado hacia la redensificación por parte de los habitantes de clase media y media-alta, afectados por el boom inmobiliario y sus consecuencias en materia de deterioro de los servicios urbanos básicos (Alba, 2009).

En el caso de Santiago se produjo impactos globalmente similares: una explosión inmobiliaria significativa y aún más perceptible por su verticalidad marcada, el alza de los precios, la aparición de dinámicas de 
gentrificación en ciertas zonas (Contreras, 2011), una mala percepción de la densidad y, al final, un deterioro marcado de la imagen urbana. Lo anterior lleva hoy a cuestionar cada vez más el proceso de redensificación (López Morales, Gasic y Meza, 2014).

Al concentrarse en la dinamización de la oferta inmobiliaria, sin considerar otras dimensiones fundamentales e indispensables para un desarrollo urbano integral, las autoridades locales de Santiago y del ex Distrito Federal no lograron impulsar ningún proceso de regeneración urbana sino más bien una dinámica de redensificación intensa y, en gran parte, insostenible.

\section{Los barrios populares de las "periferias": ¿Regeneración urbana alternativa?}

Si la regeneración urbana (tal y como se presenta en Europa y América del Norte, sobre la base de grandes proyectos urbanos en las áreas centrales de las ciudades) está todavía poco desarrollada en América Latina, existen, en cambio, experiencias de mejoramiento integral de barrios en las "periferias" ${ }^{8}$ urbanas de la región,

8 El término se refiere aquí no sólo a los espacios de urbanización más reciente, sino también a las periferias "de ayer", territorios urbanos en proceso de consolidación e integración a la ciudad, pero que siguen siendo espacios urbanos precarios. caracterizadas por la multidimensionalidad de sus objetivos y de las intervenciones realizadas (físicas, sociales, económicas, ambientales), así como por su carácter territorializado y su escala barrial. Asimismo, impulsadas por los gobiernos locales, son también ejemplos de gobernanza multi-actores en el sentido en qué suelen involucrar a actores de la sociedad civil (organizaciones sociales, y a veces, universidades) y comunidades. Siguiendo la definición genérica que se puede plantear para la regeneración urbana, estarían cumpliendo con todos sus elementos, salvo que, notablemente, los actores económicos e inversionistas privados no formarían parte del cuadro como protagonistas.

\section{LOS PROGRAMAS DE MEJORAMIENTO DE BARRIOS (PMB): ¿INICIATIVAS DE REGENERACIÓN URBANA EN LAS PERIFERIAS URBANAS?}

Los llamados Programas de Mejoramiento de Barrios, o Barrial (PMB), se han desarrollado mucho en Latinoamérica desde hace tres décadas, en particular bajo el auspicio financiero del Banco Interamericano de Desarrollo. Tal y como lo relata un exhaustivo trabajo que analiza muchos de estos proyectos, realizado para la Cámara de los Diputados en México (Orozco Martínez, 2015), los PMB consisten en una forma de solucionar los problemas de los asentamientos informales, aceptando que éstos son una realidad urbana difícil y no 
oportuna de erradicar, y que forman parte del proceso de crecimiento de las ciudades; en otras palabras, que es necesario regenerarlos y no erradicarlos. En una primera etapa, estos programas se enfocaron en la integración física de los barrios informales, construyendo vialidades y asegurando su acceso a los servicios urbanos básicos. La experiencia mostró que los beneficios de estas intervenciones físicas se veían reducidos por los problemas sociales que enfrentaban los habitantes, lo que llevó a una segunda generación de $\mathrm{PMB}$, que a partir de 1990 abordaron el problema trabajando en la integración física, social y económica de los asentamientos informales. Se trata de "atender la pobreza extrema, garantizando la integralidad con otros programas y problemas, integrando los asentamientos a la ciudad formal, reorganizando los espacios públicos y mejorando y saneando ambientalmente el barrio". Asimismo, la participación de la comunidad en todas las fases del proyecto (diseño, ejecución del mismo y mantenimiento de los servicios) suele ser parte de las características de estos proyectos (Orozco Martínez, 2015).

Los PMB han sido implementados, generalmente con mucho éxito, en numerosos países latinoamericanos (programas nacionales) y también en múltiples ciudades (programas locales). Algunos de ellos se volvieron muy conocidos, como es el caso del programa de recuperación de barrios "Quiero mi barrio" implementado en Chile desde el 2006, cuya particularidad, por el contexto chileno, es el no aplicarse en asentamientos informales sino en "vecindarios formales de pobres" (Arriagada, 2013; Ulriksen, 2019). Sin embargo, la Ciudad de Medellín, con su llamado "Urbanismo Social", constituye la experiencia probablemente más emblemática de este tipo de intervención en la región. La segunda ciudad en importancia de Colombia implementó a partir de mediados de los años 2000 esta metodología innovadora de intervención en zonas caracterizadas por altos índices de marginalidad, segregación, pobreza y violencia. Se buscó generar un proceso de consolidación barrial que permitiera estructurar y ordenar el territorio (y no solamente mejorar su accesibilidad, aunque la instalación de líneas de Metrocable fue una base esencial) a través de obras y proyectos de carácter público como equipamientos comunitarios, parques, calles, paseos y puentes peatonales para conectar los barrios, entre otros (Duque, 2015). Los Proyectos Urbanos Integrales (PUI), instrumentos de planeación e intervención física para los polígonos seleccionados, fueron una de las herramientas estratégicas de cambio, el que se logró con la participación activa de la comunidad y de la sociedad civil (Echeverri y Orsini, 2010). Si bien no se debe sacralizar lo que sucedió en Medellín entre mediados de los años 2000 y 2014, se trata de un caso muy ilustrativo de proyectos territorializados de regeneración urbana en espacios urbanos periféricos, pues éstos están 
siendo transformados y "reconstruidos sobre sí mismos", mediante intervenciones físicas, sociales y económicas (Montoya, 2014). Fue precisamente el carácter profundamente urbano del proceso de mejoramiento que llevó a denominarlo Urbanismo Social.

Otro ejemplo a menudo mencionado en materia de mejoramiento integral de barrio en la región es el Programa Favela Barrio, en Rio de Janeiro, Brasil, considerado como "una de las intervenciones más icónicas del mundo" y cuya gran continuidad (desde 1994 y ahora en su cuarta fase) es destacable (Libertun y Osorio, 2020). El Programa Hábitat, implementado en México durante la década del 2000, es otro caso de un enfoque similar en materia de intervención integral en asentamientos precarios, aunque menos conocido y más modesto (Ordóñez-Barba, Alegría-Olazábal, Mcintosh y Zenteno-Quintero, 2013).

Los ejemplos de este tipo de proyectos de intervención integral, a escala barrial, tanto urbanísticos como sociales, realizados en beneficio de los habitantes, abundan en la región y tienden a hacer de ella una referencia a nivel mundial.

\section{LA REGENERACIÓN URBANA EN EL CONTEXTO DE LOS BARRIOS DE VIVIENDA SOCIAL: UN RETO EMERGENTE EN LA REGIÓN}

Si la acepción urbanística tradicional de la regeneración urbana se refiere principalmente a los grandes proyectos de reconversión urbana realizados en las áreas centrales de las ciudades, es preciso señalar que el término suele abarcar en Europa y América del Norte también un segundo tipo de intervención urbana: la que tienen lugar en los barrios de vivienda social deteriorados. La regeneración de estos espacios urbanos constituye, de hecho, un reto mayor para las políticas urbanas en estas dos grandes regiones del mundo (Watt y Smets, 2017). Aunque no es el caso todavía en América Latina, el reto está emergiendo cada vez más donde se han construido este tipo de urbanizaciones. Si bien es cierto que la modalidad principal de producción del espacio urbano en la fase de urbanización acelerada que conoció la región a partir de mediados del Siglo XX fue, por lejos, la urbanización espontánea e informal, algunos países experimentaron, la producción de viviendas sociales "llave en mano", generalmente destinadas a sectores medios y medio-bajos de la población o a ciertas categorías de trabajadores ${ }^{9}$. Es en particular el caso de Brasil, Argentina, México y Chile, en donde se edificaron varias decenas de conjuntos habitacionales en altura a partir de los años 40 y globalmente hasta los 80 , generalmente encargados a los mejores arquitectos locales e inspirados en los principios del urbanismo moderno (García García, 2010). Una segunda generación de programas de vivienda social, mucho más masiva, se empezó a construir en

9 Entendemos aquí por "vivienda social" la vivienda terminada, adquirida por los hogares "llave en mano", que se beneficia de algún tipo de financiamiento público, sea subsidio y/o crédito. 
la región a partir de la década de los 90, en particular en Chile, país en el cual las bases de esta producción remontan en realidad al año 1978, cuando se creó el mecanismo del subsidio habitacional. Se instaló un nuevo modelo de producción, en el cual el Estado se ocupaba solamente de subsidiar a la demanda (mediante el otorgamiento a los hogares de créditos y/o subsidios), cuando la promoción inmobiliaria privada asumía la totalidad del proceso de producción. Adoptada luego por México, y también más tarde por Brasil y Colombia, la producción masiva de viviendas sociales dio lugar a la construcción de conjuntos habitacionales de gran tamaño (edificios o casas unifamiliares), física y socialmente homogéneos y ubicados en periferias lejanas (Libertun, 2017). Si los barrios de vivienda social de primera generación se vieron afectados con el paso del tiempo por un deterioro innegable, esto no fue nada en comparación con la crisis que azotó los grandes conjuntos habitacionales producidos a partir de los años 1990 y 2000. Pocos años después de ser edificados, éstos entraron en un proceso de deterioro agudo, físico y social, en particular en Chile y en México, donde empezaron a darse las primeras experiencias de esta segunda generación (Bustos-Peñafiel, 2020; Beytia, 2014; OECD, 2015).

En este contexto, las intervenciones urbanas en conjuntos de vivienda social se volvieron un nuevo reto en estos dos países latinoamericanos ${ }^{10}$. A diferencia de lo que sucede con los barrios precarios de origen informal (vivienda social "de hecho") y a pesar de la similitud en los programas implementados, las intervenciones integrales llevadas a cabo en los conjuntos formales de vivienda social en América Latina suelen ser calificadas, al igual que en Europa y América del Norte, como Regeneración Urbana. En gran parte inspiradas por la experiencia acumulada en la región en materia de mejoramiento integral de barrios, estos programas se distinguen de sus equivalentes de economías desarrolladas por estar basados, en gran medida, en un trabajo con las comunidades organizadas de vecinos y en el desarrollo social como palanca para transformar los espacios urbanos (Instituto Nacional del Fondo de la Vivienda para los Trabajadores [INFONAVIT], 2020).

Chile presenta hoy en día avances importantes frentealanuevaproblemáticallamada, demanera muy atinada, de los "con-techo" (Rodríguez y Sugranyes, 2004). Aunque se empezó a atender el problema de los condominios sociales deteriorados desde finales de los años 90, es sobre todo a partir de 2006, en el marco de la

10 Cabe señalar que Argentina es otro país de la región en el cual el reto de la regeneración urbana de los grandes conjuntos de vivienda social (en este caso, construidos antes de los 90) se está planteando ahora con fuerza y está generando reflexiones importantes acerca de las respuestas institucionales que brindarle, sea por parte del Gobierno nacional o de las autoridades locales (ver "Cómo rehabilitar los conjuntos habitacionales”, Diario Clarín, 9/9/2013). 
nueva política habitacional de mejoramiento de la calidad de vida y de la integración social, que se instaló verdaderamente la regeneración de estos barrios como tema de interés para las políticas públicas (Bustos-Peñafiel, 2020). Desde entonces, en lo que se podría calificar como un camino de experimentación, se han implementado programas de distintas índoles para atender las problemáticas muy diversas de estos barrios, incluso con un componente de demolición-reconstrucción para los casos más críticos. En la actualidad, el Ministerio de Vivienda y Urbanismo (MINVU) cuenta con un abanico de respuestas institucionales diversificado y bastante consolidado, aunque todavía en proceso de construcción. Maneja programas centrados en la atención exclusiva del universo más crítico (un poco más del 10\% del stock total de viviendas sociales), como es el caso del Programa de Regeneración de Conjuntos de viviendas sociales (iniciado en 2015), y otros, destinados a los conjuntos mejorables (Programa de mejoramiento de condominios sociales, Programa de mejoramiento de vivienda y barrio). Asimismo, se está interviniendo bajo dos modalidades muy distintas, según los casos: por medio de la atención a la demanda a través de subsidios destinados a los hogares (pilar de la política urbano-habitacional en Chile) y, en territorios específicos, a través de mecanismos de inversión. Para los casos críticos con intervenciones de re-estructuración urbana mayor (como el sector emblemático de Bajos de Mena), se están realizando planes maestros integrales de regeneración urbano-habitacional con un componente espacial y urbanístico marcado. El involucramiento de la comunidad se está buscando cada vez más en el contexto de la implementación de los programas, en particular mediante la formalización (necesaria) de organizaciones que representan a los vecinos (INFONAVIT, 2020).

Desde luego, siguen existiendo importantes retos pendientes. Sin embargo, con esta experiencia de atención a la problemática de sus condominios sociales, Chile ha entrado a formar parte del grupo de los países que, al nivel mundial, han desarrollado mayor expertise en materia de Regeneración Urbana de conjuntos de viviendas sociales, en el cual destacan en particular Francia y EEUU (OECD, 2015).

La situación de México con respecto a la necesidad de llevar a cabo intervenciones de regeneración urbana en los grandes conjuntos de vivienda social edificados a partir de la década del 2000 es más compleja. La existencia de una problemática mayor en materia de desocupación/abandono de viviendas sociales lleva a concentrar la atención sobre esta temática, sea para la elaboración de diagnósticos como para la búsqueda de "soluciones". En este contexto, no es de extrañarse que el esquema de regeneración existente haya sido hasta ahora ante todo mercantil y haya consistido en volver a vender las viviendas abandonadas a nuevos 
hogares, después de ser recuperadas por el organismo nacional financiador de créditos para acceder a la vivienda (el Instituto del Fondo Nacional de la Vivienda para los Trabajadores: INFONAVIT) y rehabilitadas por operadores privados (OECD, 2015). A diferencia de Chile, resta entonces por construir en México una verdadera política de regeneración que pueda atacar las múltiples dimensiones de la problemática con un enfoque urbanístico y que involucre al conjunto de los actores públicos, privados y de la sociedad civil.

\section{Consideraciones finales}

Descentrando la mirada, América Latina aparece como una región que posee en realidad una experiencia valiosa en materia de regeneración urbana. Ésta no tiene que ser buscada en las formas que adoptan tradicionalmente las intervenciones en este campo, que son los Grandes Proyectos Urbanos localizados en áreas centrales o en zonas de baldíos intra-urbanos. Tampoco puede ser ilustrada por los programas de revitalización de centros históricos o de redensificación de áreas centrales: éstos se asemejan mucho más a procesos que se podrían denominar, respectivamente, de puesta en valor turísticocultural y de desarrollo inmobiliario intensivo.

Las experiencias valiosas de regeneración urbana, tal y como ha sido genéricamente definida aquí esta noción, se encuentran más bien en las periferias urbanas, donde se están implementando proyectos de mejoramiento integral de barrios, inclusive ahora en barrios formales de vivienda social, como es el caso en Chile. Los modus operandi varían de un contexto a otro, pero estos proyectos comparten metodologías que toman en cuenta la problemática urbano-habitacional de manera integrada. Tratándose de iniciativas territorializadas, con un objetivo de reestructuración e integración física, social, y muchas veces económica, en beneficio de los habitantes, asociando además a diversos tipos de actores -y en particular las comunidades, estaríamos aquí ante un tipo de acción que podríamos considerar como un proceso de regeneración urbana alternativa, aunque presentando dos grandes características que la distinguen de los grandes proyectos de regeneración urbana tradicionales. Por una parte, el actor privado, central en los GPU, no es para nada el protagonista principal; al contrario, los proyectos están llevados a cabo desde las autoridades y si bien son multi-actores, es porque asocian estrechamente a organizaciones de la sociedad civil y a las comunidades. Por otra parte, la segunda diferencia mayor radica en que estos proyectos de mejoramiento integral de barrios de origen informal o formal están diseñados para mejorar las condiciones de vida y de inserción urbana de los habitantes presentes en el lugar, y no para atraer externos residentes futuros. En cambio, los grandes 
proyectos de regeneración urbana en zonas en desuso, como los programas de revitalización de centros históricos y de redensificación/ repoblamiento de áreas céntricas, buscan, casi siempre, en América Latina, regenerar los espacios urbanos para el beneficio de habitantes futuros que forman parte de una clase media-alta gentrificadora, en gran medida idealizada (Betancur, 2014). Si bien es cierto que los proyectos de mejoramiento integral de barrios también pueden llegar a conllevar impactos en materia de transformación ulterior de su población residente, este tipo de recomposición social no forma parte de los objetivos de la intervención urbana, sino que es una consecuencia no controlada de ésta.

Por estas diferencias fundamentales, resultaría importante cambiar la mirada sobre estas experiencias de mejoramiento integral de barrios, viéndolas de ahora en adelante como lo que son en realidad: estrategias de regeneración urbana virtuosas, y no la simple expresión de un urbanismo de regularización, de ajuste de cuentas y "saldo de deuda" con los más pobres. Considerar de esta forma a los proyectos de mejoramiento integral de barrios (que ya son ejemplo para otras regiones del mundo) podría contribuir a rehabilitar en América Latina la noción urbanística de regeneración urbana, que tiende a ser considerada hoy con sospecha, como un proceso de producción urbana estrechamente asociado a un urbanismo "ligado a los promotores" (Cuenya, 2012). Atreviéndonos a soñar con un mundo urbanístico mejor, más allá de mostrar experiencias exitosas realizadas en beneficio de las comunidades, los proyectos de mejoramiento integral de barrios podrían llegar a ser fuentes de inspiración para la elaboración de propuestas alternativas para concebir grandes proyectos urbanos en las áreas centrales de las ciudades en beneficio de sus habitantes, es decir de estratos socioeconómicos medio-bajos y bajos, y no de interés económicos ajenos.

En el contexto de los objetivos por lograr una mayor sostenibilidad urbana, la regeneración urbana como proceso de reconstrucción de la ciudad sobre sí misma es, y seguirá siendo, imprescindible en las urbes, en particular en Latinoamérica. Rehabilitarla como un proceso urbano posiblemente virtuoso y no enfocado hacia lógicas neoliberales y de mercado, dándole un nuevo impulso alternativo, constituiría un reto de alcance mayor.

\section{Referencias bibliográficas}

Aguayo, A. (2016). Nuevo Polanco: renovación urbana, segregación y gentrificación en la Ciudad de México. Iztapalapa. Revista de Ciencias Sociales y Humanidades, 37(80), 101-123.

https://doi.org/10.28928/revistaiztapalapa/802016/ atc4/aguayoayalaa

Alba, M. d. (2009). Representaciones y prácticas sociales en torno a políticas urbanas: la movilización NIMBY frente a la redensificación de 
las zonas centrales de la Ciudad de México. Cultura y representaciones sociales, 3(6), 43-72.

Amirtahmasebi, R., Orloff, M., Wahba, S., y Altman, A. (2016). Regenerating urban land: A practitioner's guide to leveraging private investment. Washington, DC: Banco Mundial. Recuperado de https://openknowledge.worldbank.org/ handle/10986/24377

Arriagada, C. (2013). Regeneración focalizada en integración social de vecindarios formales de pobres en grandes ciudades chilenas. Cambridge (EEUU): Lincoln Institute of Land Policy.

Audirac, I. (2014). Shrinking cities in Latin America: an oxymoron? En H. Richardson y C. Woon Nam, Shrinking cities: a global perspective (pp. 28-46). Londres: Routledge.

Aureau, E., Dufourmantelle, A., y Narring, P. (2015). Regards sur les grands projets urbains en Europe. Paris: Consejo General del Medio Ambiente y del Desarrollo Sostenible.

Betancur, J. (2014). Gentrification in Latin America: overview and critical analysis. Urban Studies Research, article id 986961. https://doi.org/10.1155/2014/986961

Beytia, P. (2014). Barrios de vivienda social: tres focos para revertir su deterioro urbano. Santiago: Instituto de Políticas Públicas UDP.

Bustos-Peñafiel, M. (2020). Desafíos para enfrentar el deterioro de una producción cuantitativa. Vivienda social en copropiedad en Chile. Bitácora Urbano Territorial, 30(3), 247-261. https://doi.org/10.15446/bitacora.v30n3.86821
Cabrera, N. (2019). Gentrificación en áreas patrimoniales latinoamericanas: cuestionamiento ético desde el caso de Cuenca, Ecuador. urbe, revista brasileira de gestão urbana, 11. https://doi.org/10.1590/2175-3369.011.e20180201

Carrión, F. (2017). Centros históricos: ¿es posible y necesario el espacio residencial en su seno? En A. Pineda, y M. Velasco (Coords.), Ciudades y centros históricos. Los retos de la vivienda y la habitabilidad (pp. 21-33). México: PUEC.

CEPAL. (2018). Plan de acción regional para la implementación de la nueva agenda urbana en América Latina y el Caribe, 2016-2036. Santiago de Chile: CEPAL.

Chaline, C. (1996). Les villes nouvelles dans le monde. Paris : Presses Universitaires de France.

Chasseriau, A. (2002). Les grands équipements au service de la régénération urbaine : l'exemple de la métropole de Nantes Saint-Nazaire. ESO Travaux et documents, (18), 7-16.

Coing, H. (1966). Rénovation urbaine et changement social: l'îlot no 4 (Paris 13e). Paris: Editions Ouvrieres.

Contreras, Y. (2011). La recuperación urbana y residencial del centro de Santiago: Nuevos habitantes, cambios socio espaciales significativos. EURE, 37(112), 89-113. https://doi.org/10.4067/S0250-71612011000300005

Cortés Chavez, S. (2009). La capacidad de carga como herramienta para la ordenación sostenible del territorio. Cuaderno de Investigación Urbanística, (65), 35-56. 
Crossa, V. (2009). Resisting the entrepreneurial city: Street vendors' struggle in México city's historic center. International Journal of Urban and Regional Research, 33(1), 43-63.

https://doi.org/10.1111/j.1468-2427.2008.00823.x

Cuenya, B. (2012). Grandes proyectos urbanos: miradas críticas sobre la experiencia argentina y brasileña. Buenos Aires: Café de las Ciudades.

De Magalhães, C. (2015). Urban regeneration. En J. D. Wright (Ed.), International Encyclopedia of the Social \& Behavioral Sciences (2a ed., pp. 919-925). Oxford: Elsevier. https://doi.org/10.1016/B978-0-08-097086-8.74031-1

Delgadillo, V. (2008). Mejoramiento habitacional en las áreas urbanas centrales de América Latina. Del combate de tugurios a la rehabilitación habitacional progresiva. Revista INVI, 23(63), 89-120.

http://revistainvi.uchile.cl/index.php/INVI/ article/view/444

Delgadillo, V. (2016). Ciudad de México, quince años de desarrollo urbano intensivo: la gentrificación percibida. Revista INVI, 31(88), 101-129. https://doi.org/10.4067/S0718-83582016000300004

Duque, I. (2015). La cultura como estrategia de transformación y promoción urbana en Bogotá y Medellín. Revista de Geografía Norte Grande, (61), 25-43.

https://doi.org/10.4067/S0718-34022015000200003

Echeverri, A. y Orsini, F. (2010). Informalidad y urbanismo social en Medellín. En M. Hermelin, A. Echeverri y J. Giraldo (Eds.), Medellin medio ambiente urbanismo y sociedad. Medellín: Universidad EAFIT.

García García, E. (2010). Documentos de arquitectura moderna en América Latina 1950-1965. Volumen 4: Vivienda social en Argentina, Brasil, Chile y México. Madrid: Gobierno de España, Ministerio de Ciencia e Innovación.

González Couret, D. (2015). Tendencias actuales de la arquitectura y el urbanismo en América Latina. 1990-2014. Revista científica de arquitectura y urbanismo, 36(2), 128-139.

Hernández Cordero, A. (2012). El proyecto Alameda. Ciudades, (95), 32-38.

Hidalgo, R., Borsdorf, A., y Zunino, H. (2008). Las dos caras de la expansión residencial en la periferia metropolitana de Santiago de chile: precariópolis estatal y privatópolis. En R. Hidalgo y P.C. Pereira (Eds.), Producción inmobiliaria y reestructuración metropolitana en América Latina (pp. 167-196). Sao Paulo: Universidad de Sao Paulo, Facultad de Arquitectura y Urbanismo.

Instituto Nacional del Fondo de la Vivienda para los Trabajadores. (2020). Regeneración de conjuntos de vivienda social en México. Una visión prospectiva. México: INFONAVIT.

Jajamovich, G. (2012). De Parque España a Puerto Madero. Proyectos urbanos y gestión entre Argentina y España". En B. Cuenya, Grandes proyectos urbanos: miradas críticas sobre la experiencia argentina y brasileña (pp. 119-144). Buenos Aires: Café de las Ciudades.

Jajamovich, G. (2016). Puerto Madero 'en movimiento': movilidad de políticas y modelos urbanos 
en América Latina (1999-2012). Revista INVI, 31(87), 59-84.

https://doi.org/10.4067/S0718-83582016000200002

Jajamovich, G. (2019). Grandes proyectos urbanos alternativos o alternativas a los grandes proyectos urbanos: una revisión a partir del concepto de just city. Cuadernos de Geografía: Revista Colombiana de Geografía, 28(2), 349-407. https://doi.org/10.15446/rcdg.v28n2.72090

Kozak, D. y Feld, N. (2018). Grandes proyectos urbanos y su relación con la ciudad: el caso de Puerto Norte (Rosario, Argentina). EURE, 44(133), 187-210.

https://doi.org/10.4067/s0250-71612018000300187

Libertun, N. (2017). ¿Por qué allí? Los motivos por los que los promotores privados de vivienda social construyen en las periferias de las ciudades de América Latina. Washington: Banco Interamericano de Desarrollo.

Libertun, N. y Osorio, R. (2020). Bairro, 10 años después, 2020. Washington: Banco Interamericano de Desarrollo, $49 \mathrm{p}$.

López Morales, E., Gasic, I. y Meza, D. (2014). Actores sociales y políticos contestando un modelo de urbanismo pro-empresarial: el lado B de la renovación urbana de Santiago de Chile. XIII Coloquio Internacional de Geo-crítica "El control del espacio y los espacios de control", Barcelona.

http://www.ub.edu/geocrit/coloquio2014/Ernesto\%20 Lopez\%20Morales.pdf

Lungo, M. (2005). Grandes proyectos urbanos. Una vision general. Urbana, 10(37), 16-43.
Merlin, P. (2015). Introduction. En P. Merlin y F. Choay (Eds), Dictionnaire de l'aménagement et de l'urbanisme. Paris : Presses Universitaires de France.

Montoya, N. (2014). Urbanismo social en Medellín: una aproximación a partir de la utilización estratégica de los derechos. Estudios Politicos, (45). 205-222.

Navarrete, D. (2017). Turismo gentrificador en ciudades patrimoniales. Exclusión y transformaciones urbano-arquitectónicas del patrimonio en Guanajuato, México. Revista INVI, 32(89), 61-83. https://doi.org/10.4067/S0718-83582017000100061

Orozco Martínez, Y. (2015). Hacia políticas de urbanización integral: los programas de mejoramiento de barrios en América Latina. México: Centro de Estudios Sociales y de Opinión Pública de la Cámara de Diputados.

Paquette, C. (2008). El Metrobús en el contexto de la redensificación urbana: implicaciones y oportunidades. En J.L. Lezama y C. Salazar, Construir ciudad. Un análisis multidimensional para los corredores de transporte en la Ciudad de México (pp. 195-240). México: El Colegio de México.

Paquette, C. (2014). Las políticas de inversión urbana en América Latina. En C. Quenan y S. Velut (Eds.), Los desafíos del desarrollo en América Latina. Dinámicas socio-económicas y políticas públicas (pp. 185-211). Paris: Agencia Francesa de Desarrollo/Instituto de las Américas.

Pineda, A. y Velasco, M. (Coords.). (2017). Ciudades y centros históricos. Los retos de la vivienda y la 
habitabilidad. México: Programa Universitario de Estudios de la Ciudad.

OECD. (2015). OECD territorial reviews: Valle de México, México. Paris, OECD. https://doi.org/10.1787/9789264245174-en

Ordóñez-Barba, G., Alegría-Olazábal, T., Mcintosh, C., y Zenteno-Quintero, R. (2013). Alcances e impactos del Programa Hábitat en comunidades pobres urbanas en México. Papeles de Población, 19(77), 231-267.

Roberts, P. (2008). The evolution, definition and purpose of urban regeneration. En P. Roberts y H. Sykes, Urban regeneration: A Handbook (pp. 9-36). Londres: Sage.

Rodrigues-Malta, R. (2001). Régénération urbaine : variations sud-européennes. L'Information géographique, 65(4), 321-339.

https://doi.org/10.3406/ingeo.2001.2774

Rodríguez, A., y Sugranyes, A. (2004). El problema de vivienda de los "con techo. EURE, 30(91), 53-65.

https://doi.org/10.4067/S0250-71612004009100004

Sedrez, L. (2013). Naturaleza urbana en América Latina. Ciudades diversas y narrativas comunes. RCC Perspectives, (7),59-66. https://doi.org/10.5282/rcc/5922

Stamm, C. (2008). Commerce de rue et politiques publiques dans les centres historiques. Expulsion, relocalisation et résistances à Mexico et Lima. Autrepart, (45), 91-104.

Tamayo, S. (Coord.). (2007). Los desafíos del bando 2. Evaluación multidimensional de las políticas habitacionales en el DF, 2000-2006. México: Seduvi-Invi-Uacm-Cam.

Ulriksen, C. (2019). Genealogía del primer programa chileno de recuperación de barrios vulnerables "Quiero mi Barrio" en su primera generación 2006-2010. Revista INVI, 34(96), 9-50. https:// doi.org/10.4067/S0718-83582019000200009

Valenzuela, A. (2007). Santa Fe (México): Megaproyectos para una ciudad dividida. Cuadernos Geográficos, 40(1), 53-66.

Watt, P. y Smets, P. (Eds.). (2017). Social housing and urban renewal: A cross-national perspective. Bingley: Emerald Publishing. 


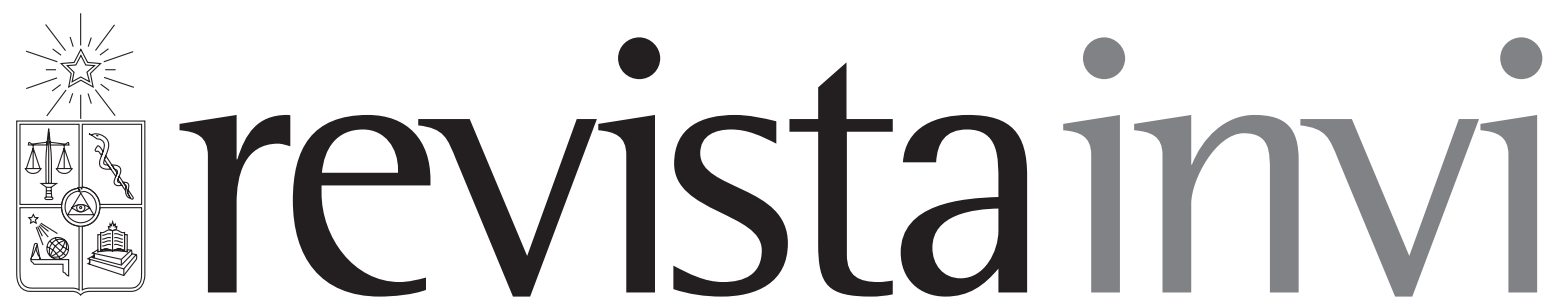

Revista INVI es una publicación periódica, editada por el Instituto de la Vivienda de la Facultad de Arquitectura y Urbanismo de la Universidad de Chile, creada en 1986 con el nombre de Boletín INVI. Es una revista académica con cobertura internacional que difunde los avances en el conocimiento sobre la vivienda, el hábitat residencial, los modos de vida y los estudios territoriales. Revista INVI publica contribuciones originales en español, inglés y portugués, privilegiando aquellas que proponen enfoques inter y multidisciplinares y que son resultado de investigaciones con financiamiento y patrocinio institucional. Se busca, con ello, contribuir al desarrollo del conocimiento científico sobre la vivienda, el hábitat y el territorio y aportar al debate público con publicaciones del más alto nivel académico.

Director: Dr. Ricardo Tapia Zarricueta, Universidad de Chile, Chile.

Editor: Dr. Luis Campos Medina, Universidad de Chile, Chile.

Editor asistente: Dr. Walter Imilan, Universidad de Chile, Chile.

Coeditora: Srta. Sandra Rivera, Universidad de Chile, Chile.

\section{COMITÉ EDITORIAL:}

Dr. Victor Delgadillo, Universidad Autónoma de la Ciudad de México, México.

Dra. María Mercedes Di Virgilio, CONICET/ IIGG, Universidad de Buenos Aires, Argentina.

Dra. Irene Molina, Uppsala Universitet, Suecia.

Dr. Gonzalo Lautaro Ojeda Ledesma, Universidad de Valparaíso, Chile.

Dra. Suzana Pasternak, Universidade de São Paulo, Brasil.

Dr. Javier Ruiz Sánchez, Universidad Politécnica de Madrid, España.

Dra. Elke Schlack Fuhrmann, Pontificia Universidad Católica de Chile, Chile.

Dr. Carlos Alberto Torres Tovar, Universidad Nacional de Colombia, Colombia.

Sitio web: http://www.revistainvi.uchile.cl/

Correo electrónico: revistainvi@uchilefau.cl

Licencia de este artículo: Creative Commons Atribución-Compartirlgual 4.0 Internacional (CC BY-SA 4.0) 